\title{
CONOCIMIENTOS TRADICIONALES EN LA PRODUCCIÓN DE PLANTAS MEDICINALES PARA EL DESARROLLO PRODUCTIVO SOSTENIBLE EN CHAGUITILLO, MATAGALPA 2011-2013
}

Jossarys Massielle Gazo Robles

\section{Resumen}

El presente estudio tiene el propósito de analizar desde un enfoque antropológico la producción de plantas medicinales a través del uso de la hidroponía. Según las investigaciones, en la comunidad de Chagüitillo existen plantas nativas con características curativas, además se encuentran dos empresas hidropónicas: "Hidropónicas de Nicaragua S.A y HerbayFresh S.A", siendo este contexto por lo que seleccionó esta zona. La investigación estructura los beneficios naturales de cada una de las especies medicinales necesarias para el análisis y determina cuál de las especies es la más viable para su producción a través del método hidropónico. Se plantea el uso de la técnica de cultivo hidropónico como alternativa de conservación del medio ambiente y de creación de huertos hidropónicos como parte de la estrategia del desarrollo sostenible. La investigación utilizó las variables: hidroponía, plantas medicinales, conocimiento tradicional, etno ecología y etno botánica. La metodología antropológica comprende cuatro fases: reconocimiento del área, delimitación del área de estudio, elaboración, realización de observaciones de campo de tipo participativas, aplicación de las entrevistas y encuestas. Por último el procesamiento, análisis y validación de la información. Se concluye que el conocimiento tradicional, la experiencia y la sabiduría forman la base de la etnobotánica que incide en la aplicación de los saberes con fines utilitarios, dinámicos y relacionales, especialmente en cuanto a la producción de plantas medicinales con base en el método hidropónico. La innovación con estos tipos de prácticas han generado un impacto positivo y ecológicamente amigable del entorno ambiental y en la economía de las/los productoras/es.

Palabras claves: hidroponía, plantas medicinales, conocimiento tradicional, etno ecología y etnobotánica.

\begin{abstract}
This research aimed to analyse through an anthropological approaches herbal production by using hydroponics techniques. According to research, there are native plants at Chagüitillo community that have healing properties, besides found two hydroponic enterprise "Hidropónicas de Nicaragua S.A y HerbayFresh S.A", reason that this area was selected. The study defined natural benefits of each herbal species necessary to analyse and determine which are the most viable to crop through hydroponic methods. Hydroponics technics proposed as an alternative for environmental conservation, as well as the establishment of hydroponics gardens as a sustainable development strategy. The research variables: hydroponics, herbal, native knowledge ethno-ecology, ethnobotany. Anthropological method comprises four phases: area recognition, study area delimitation, field observation with participatory methodologies, interviews and surveys. Finally, data processing analysis and validation. Conclusion were that native knowledge, experience and wisdom form the basis of ethnobotany influences application of knowledge with utilitarian, dynamic and relational purposes, especially regards hydroponic production of herbals. This innovative technique have generated an environmentally friendly impact and positive impact regarding to producers economy.
\end{abstract}

Key words: hydroponics, herbals, native knowledge, ethno-ecology, ethnobotany. 


\section{Introducción}

Eato investigación es resultado análisis, desde un enfoque de plantas medicinales por medio del uso de la hidroponía. La finalidad es la identificación de los beneficios naturales de cada una de las especies medicinales nativas seleccionada en dicha comunidad.

Las plantas medicinales históricamente se han usado para prevenir y combatir casi todo tipo de enfermedades. Sin embargo con el auge de la medicina fármacoquímico se ha disminuido su uso, hasta el extremo de estar en peligro muchas prácticas ancestrales, populares en épocas anteriores.

La investigación se desarrolló en la comunidad de Chagüitillo dado que en este lugar había posibilidades de implementar la técnica hidropónica como alternativa para cultivar especies medicinales. Además de incentivar la promoción de los conocimientos tradicionales.

El objetivo general se buscaba analizar desde un enfoque antropológico la producción de plantas medicinales como alternativa de conservación del conocimiento tradicional y utilitario; la caracterización (social, económica, políticas y cultural) de la población de Chagüitillo, Sébaco- Matagalpa; la identificación de las especies medicinales nativas y la implementación de la técnica hidropónica en la búsqueda de alternativas para la diversificación productiva de los patios.

\section{Aspectos Conceptuales}

Se consideró necesaria la definición conceptual de algunos términos que permitieran guiar el trabajo. El aspecto de la Hidroponía fue estudiado a partir de la propuesta que hace Laura Alpizar Antillon, "Hidroponía: Cultivo sin tierra". Las plantas medicinales son definidas por Fernando Muños (1996) en su libro "Plantas Medicinales y Aromáticas". Otros conceptos definidos fueron: medicina tradicional, producción alimentaria, modo de producción y trabajo.

Dentro de la teoría antropológica se abordó, la etno ecología, que pone el acento en la sabiduría de las poblaciones frente a los conocimientos científicos Así lo explica Toledo:

La sabiduría se basa en la experiencia concreta y en las creencias compartidas por los individuos acerca del mundo circundante y conservada mediante testimonios. Los saberes locales son sistemas de conocimiento holísticos, acumulativos, dinámicos y abiertos, que se construyen con base en las experiencias locales transgeneracionales $\mathrm{y}$, por lo tanto, en constante adaptación a las dinámicas tecnológicasysocioeconómicas(Toledo, 2008, pág. 101).

La Etnobotánica, fue otro elemento teórico definido en esta investigación. Según Richard Evans Schultes (1990) es el estudio de las sabidurías botánicas tradicionales. En otras palabras, es la forma en que las plantas son utilizadas por el hombre, así como las técnicas que de ello se derivan hasta integrarlas en su patrimonio cultural.

Se retomaron los aportes teóricos de Víctor M. Toledo. Por medio de este planteamiento se identificó la importancia ecológica de los saberes tradicionales, a través del conocimiento relacional, utilitario y dinámico, permitiendo distinguir las cosmovisiones indígenas sobre los elementos que permiten los procesos de producción de plantas medicinales. 
Otro elemento teórico importante utilizado, fue la definición de Ecología Cultural, planteada por Julián Steward. Este autor alude a tres aspectos: el análisis de métodos de producción en el entorno, el modelo de comportamiento humano, y la relación de las técnicas de producción con los otros elementos de la cultura. Su teoría "Los modos de interactuar con el entorno moldean los modos de comportamiento", son retomados al estudiar la realidad de Chagüitillo, fundamentalmente al relacionar el uso que las personas le dan a la naturaleza marcando su realidad.

A partir de la definición de los enfoques teóricos antropológicos, se plantea que la ciencia occidental se ha nutrido de saberes locales. En el caso de la comunidad de Chagüitillo se han utilizado algunas plantas medicinales para la creación de huertos familiares, a base de la nueva técnica de hidroponía, haciendo que en ese espacio cultural confluyan y dialoguen visiones y prácticas que dialogan diversas y/o heterogéneas.

\section{Métodos y Técnicas}

El estudio se caracterizó por tener un enfoque de investigación mixta, es decir recurre a las técnicas de investigación cualitativa, característica de la antropología y del método cuantitativo. El tipo de investigación que se realizó fue descriptivo y explicativo sometido a un proceso de análisis de cada una de la variables manifestada en diversos escenarios y relacionados en determinado momento, con el objeto de estudio (la producción de plantas medicinales con tecnología hidropónica y su entorno cultural), donde se especifican y miden cada una de estas, de acuerdo a lo que se pretende trabajar.

\section{Población y muestra}

En la comunidad de Chagüitillo se tomó la muestra probabilística y no pirobalística (muestreo por cuotas) definida en base a criterio de selección, aquellos pobladores que ejercen actividades económicas (productores), así como líderes (casta indígena) y las personas que consumen plantas medicinales.

\section{Método cualitativo y método cuantitativo}

El enfoque cualitativo, utilizó el método etnográfico, la entrevista, observación directa y la participativa, diario de campo, observación directa y participante. Se utilizó el análisis de contenido, dando paso a la lectura y re-lectura de cada entrevista, contrastadas en algunos casos por la observación participante. Seguidamente fueron codificadas y separadas en unidades de significados colectivos y palabras representativas las cuales dieron lugar a los pre-temas.

Desde el enfoque cuantitativo se aplicaron instrumentos de tipo de encuestas, usando la escala de Likert. El análisis de los datos se realizó utilizando la estadística descriptiva del software Microsoft Excel (2006).

La investigación comprende cuatro fases

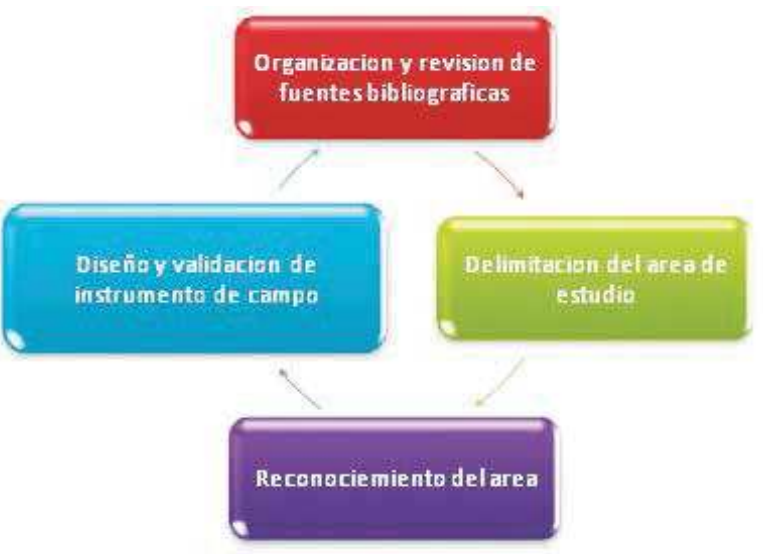




\section{Resultados}

\section{Ubicación geográfica de la zona de estudio}

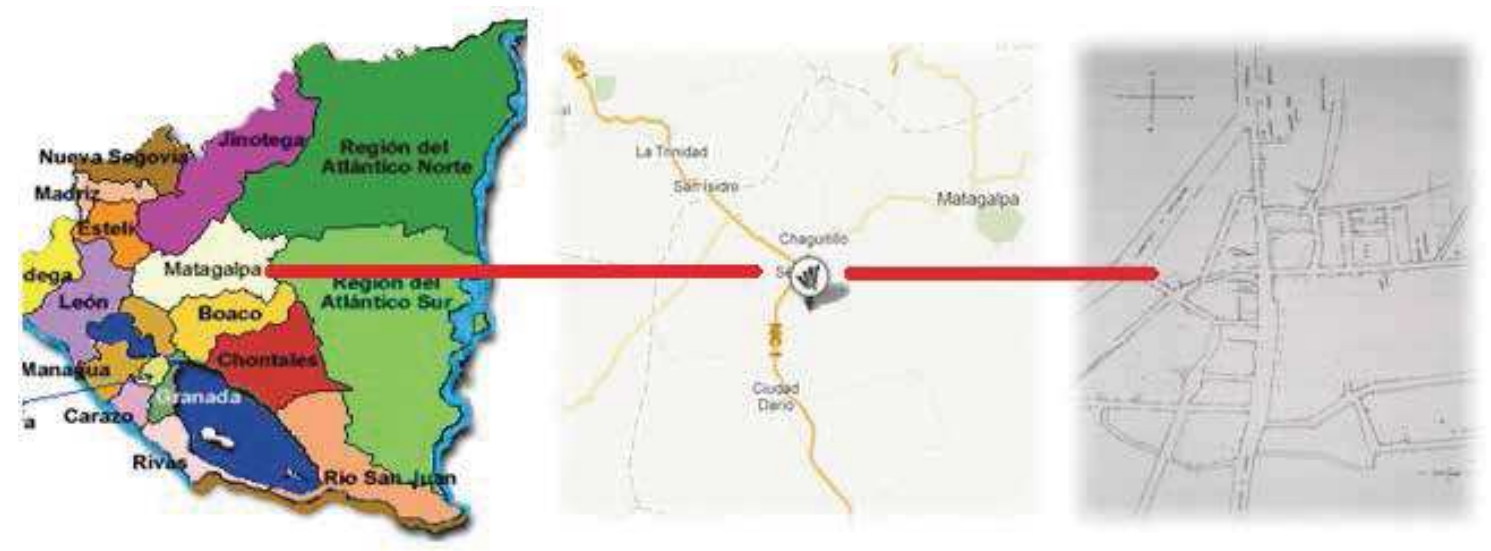

El estudio fue realizado en la comunidad de Chagüitillo, ubicada en el km 107 de la carretera Managua - Matagalpa y a 3 $\mathrm{km}$ al Norte del municipio de Sébaco. La comunidad Villa Chagüitillo, se encuentra a una altitud entre 480 a $520 \mathrm{msnm}$. Limita al Norte con la comunidad el Cacao, al Sur con Frailes, al Este con las Palomas y al Oeste con Tierras Blancas.

Chagüitillo fue un asentamiento prehispánico de alta densidad poblacional donde se desarrolló una gran cultura precolombina. Esta herencia cultural se observa en sus abundantes vestigios preculturales encontrados como: la cerámica pre-colombina decorada, numerosos montículos (ruinas indígenas) y sus impresionantes obras del arte rupestre (pictografías).

Chagüitillo cuenta con un Centro Cultural, "Domingo Sánchez Salgado", con una biblioteca y un museo precolombino. Estos centros fueron creados por la Asociación para el Desarrollo de la Comunidad de Chagüitillo) $(\mathrm{ADCH})$, bajo el mandato de la profesora Cándida Miranda.

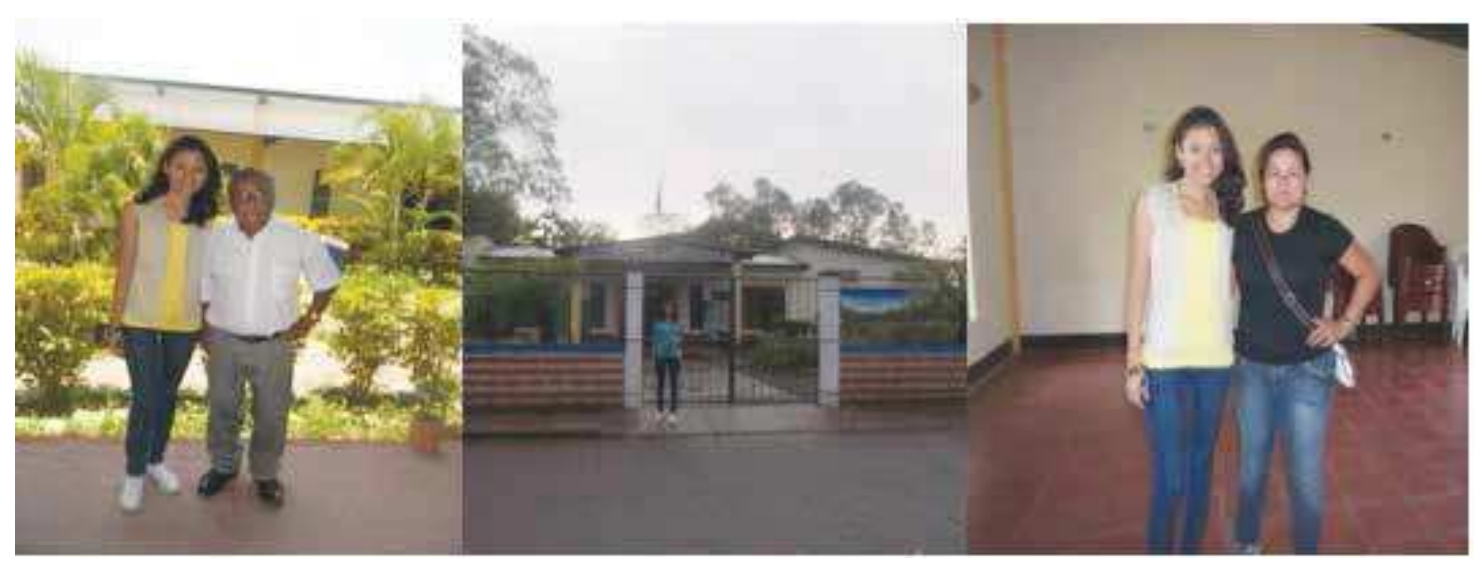

Lic. José Vicente Salazar. Responsable de proyectos y Lic. Victoria María Rayos 
El encargado de la Gestión del Proyecto ADH, don José Vicente Salazar, recuerda que: $\mathrm{ADCH}$ obtuvo su personería jurídica ante el consejo de estado en 1984, desde entonces se ha convertido en máxima entidad responsable del fomento al desarrollo de la Infraestructura turística y cultural de Villa Chagüitillo. Hagestionado proyectos en pro de la educación, vivienda, cultura, deportes, porque su objetivo es

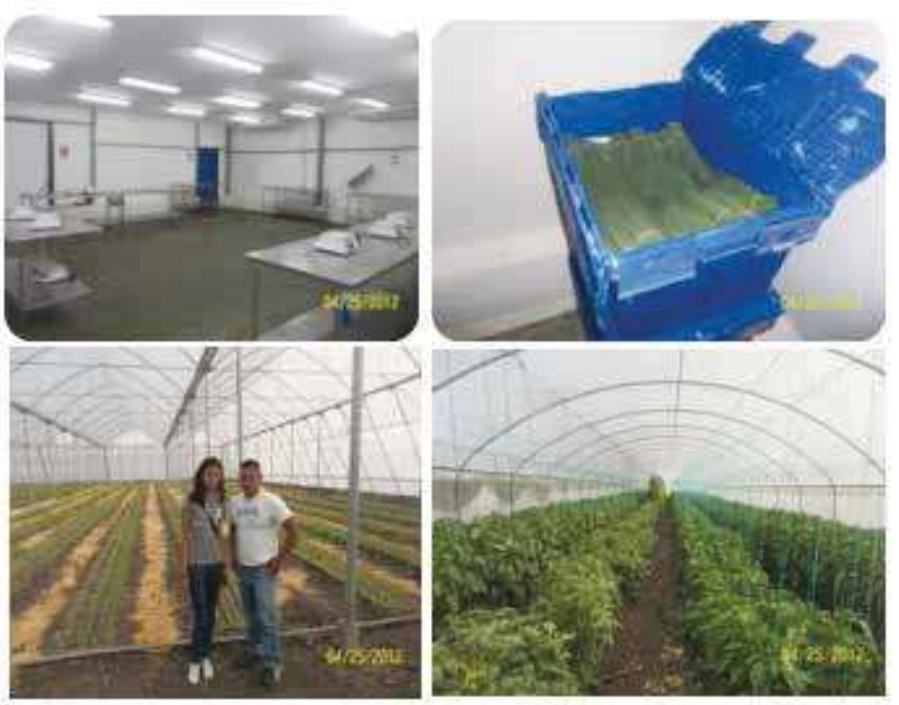
aumentar las inversiones por medio del fortalecimiento y el acondicionamiento de las pequeñas rutas turísticas comunitarias del Valle de Sébaco, combinando los sitios arqueológicos de arte rupestre indígena con las actividades eco turísticas (Vicente Salazar, 24/04/2012).

\section{Desarrollo de la hidroponía en Chagüitillo - Sébaco, Matagalpa.}

En el Valle de Chagüitillo, la técnica y producción hidropónica llega en el 2005, con establecimiento de la primera fábrica Hidropónicas de Nicaragua, S.A. gerenciada por el el señor Manolo Porro. Esta empresa nicaragüense-israelita estaba bajo el régimen de zona franca, Horti-Tech, la cual se dedicaba a la producción y exportación de chiltoma, con buena recepción en el mercado estadounidense, cuyo comprador único y potencial, Matronardi, es una empresa con 50 años de experiencia.

En el 2010, llega a la comunidad la segunda Zona Franca Hidropónica denominada Valley Fresh Herbs. S.A, administrada por Bayardo José Flores Duarte. En esta plantas medicinales como Cebollín y Albahaca utilizando técnicas y métodos israelitas. Las semillas utilizadas para la producción son compradas en Granada.

Estas empresas bajo régimen de zona franca, han promovido el método de cultivo hidropónico porque les permite cultivar plantas con un consumo reducido de agua y esfuerzos físicos menores. Bajo esta concepción técnica y metodológica de producción se puede producir hortalizas frescas, sanas en abundancia, lo cual permite la competencia en el exigente mercado internacional.

Un elemento innovador es la hidroponía en la comunidad de Chagüitillo, técnica conocida por la población. Al retomar el aspecto de la adquisición del conocimiento se puede dividir en dos aspectos: personas que han adquirido su conocimiento empíricamente, al escuchar a otros miembros de su familia o amigos; otras que son ingenieros agrónomos y han trabajado en las hidropónicas, donde han aplicado sus conocimientos o los han adquirido por su desempeño laboral. 
Según Víctor Ramírez Romero:

Lahidroponía es proceso de cultivar con sustratos que no necesitan tierra, por eso hidra de agua. La hidroponía sirve para cultivar sin insecticidas y ahorrar dinero. Además es un generador de empleo. Mis conocimientos sobre hidroponía los obtuve hace seis años, al comenzar a trabajar en la Hidropónica de Nicaragua S.A. A partir de hace tres años, comencé a crear mi huerto en el patio de la casa, los cultivos que produzco, chiltoma y coliflor, sirven de autoconsumo. Me inicie en el método hidropónico para experimentar. Las plantas a través del método hidropónico se alimentan a través del sustrato, en mi caso estoy probando con la fibra de coco, esto lo están haciendo donde trabajo, de ahí lo retomo para mi huerto." (Ramírez, 23/04/2012)

Plantas medicinales nativas de Chagüitillo Para explicar la producción de las plantas medicinales por medio de la hidroponía, es importante reconocer las plantas medicinales nativas de Chagüitillo, especies promisorias alternativas de diversificación productiva.

Las plantas medicinales, son aquellos vegetales que elaboran unos productos llamados principios activos, sustancias que ejercen una acción farmacológica, beneficiosa o perjudicial, sobre el organismo vivo. Su utilidad primordial, a veces específica, es servir como droga o medicamentos que alivie la enfermedad o restablezca la salud perdida. Esto es afirmado por Evelio Palacios, Ing. agrónomo y miembro de la comunidad indígena: "Todo árbol que al quitarle la corteza sea roja, posee características curativas" (Palacios, Evelio 25/04/2012)

Según Néstor Dávila:

La Comunidad indígena de Chagüitillo se caracteriza, por poseer especies de genízaro, tigüilote, zarza, espina blanca, laurel, madero negro, matapalos. Los recursos naturales de Villa Chagüitillo caracterizado por poseer bosques de madera, ha sufrido despale indiscriminado, reduciendo las poblaciones de especies como es el caso específico el madero negro. (Davila, 2012)

Las siguientes plantas medicinales son nativas de la zona: carao, guapinol, genízaro y jiñocuabo. Según Juan Palacios, ex - presidente de la comunidad indígena, son plantas grandes, de las que se utiliza la cascara para los cocimientos. También existen plantas de porte pequeño tales como "el apazote, dormilona, limón, orégano, pico de pájaro, llantén, sábila y uña de gato". (Palacios G, 2012). 


\section{Cuadro №1. Plantas medicinales nativas de Chagüitillo}

\begin{tabular}{|c|c|c|}
\hline $\begin{array}{l}\text { PLANTA } \\
\text { MEDICINAL }\end{array}$ & IMAGEN & PROPIEDAD CURATIVA \\
\hline APAZOTE & & $\begin{array}{l}\text { Sirve para el sistema digestivo (parte aérea): dolor de estómago, indigestión, diarrea, } \\
\text { parásitos, lombrices, amebas. Además sirve para la piel: hongos, llagas, ulceras } \\
\text { cutáneas, pajuelillas. }\end{array}$ \\
\hline CARAO & & $\begin{array}{l}\text { Ayuda al sistema respiratorio: contra la fiebre, catarro, tos, tosferina. Estimula el } \\
\text { apetito, calma el dolor de estómago y la diarrea. Combate la infección uninaria. Ayuda } \\
\text { a regular la menstruación. Elimina ronchas, paño, hongos, salpullidos, herpes, } \\
\text { sarampión. Elimina la anemia, purifica la sangre. }\end{array}$ \\
\hline DORMILONA & & $\begin{array}{l}\text { M. Púdica L. Parásitos, inflamaciones Diarrea, Vómito, etc. Su principal substancia } \\
\text { activa, es un la Curcina, un alcaloide que causa desde debilidad ceguera. Se } \\
\text { recomienda sólo en adultos. }\end{array}$ \\
\hline GUAPINOL & & $\begin{array}{l}\text { El principal uso medicinal que se le da al guapinol, es contra la tos además de otros } \\
\text { trastornos respiratorios como gripe y asma. También es utilizada en desórdenes de } \\
\text { tipo digestivo como disenteria, diarrea y úlcera. En padecimientos renales, cuando hay } \\
\text { inflamación de los rîhones, se prepara un cocimiento de esta planta junto con oreja de } \\
\text { lobo (Clusas), bromilla, bejuco de tres costillas, ruda, guarumbo (Cecropiaobtusifolia), } \\
\text { cabello de elote bejuco guaco (Aristolochiasp), anona (Annonareticulata), y cola de } \\
\text { caballo (Equisetummyriochaetum); se toma como agua de uso.P }\end{array}$ \\
\hline GENIZARO & & $\begin{array}{l}\text { Quitar dolor de cabeza. Tiene hojas compuestas, bipinnadas de } 3-9 \text { pares de hasta } 1 \\
\text { dm de largo, de } 2-4 \mathrm{dm} \text { de longitud, raquis piloso, y las flores, de color rosado, se } \\
\text { reúnen en inflorescencias vistosas situadas al final de las ramitas. Los frutos son } \\
\text { legumbres o vainas oscuras de } 8 \text { a } 20 \mathrm{~cm} \text { de largo. Se utiliza como forrajera por sus } \\
\text { legumbres verdes y por sus semillas que son comestibles. También se cultiva como } \\
\text { omamental. }\end{array}$ \\
\hline JIN̂OCUABO & & $\begin{array}{l}\text { La corteza cocida se usa como antipirético, las hojas maceradas en agua contra el } \\
\text { salpullido y como purgante. El agua tibia o caliente de la corteza cocida con sal es un } \\
\text { buen antiespasmódico y sirve también como desinflamatorio aplicándola con paños en } \\
\text { partes del cuerpo afectadas por golpes o dislocaciones. }\end{array}$ \\
\hline LIMÓN & & $\begin{array}{l}\text { Resfrió, gripe, tos rebelde, calentura, amigdalitis, dolor de estómago, acidez, diarrea, } \\
\text { facilita el parto, expulsa la placenta, }\end{array}$ \\
\hline LLANTEN & & $\begin{array}{l}\text { Ayuda contra la faringitis, laringitis, amigdalitis, higado, diarrea infecciosa, gastritis, } \\
\text { ulcera gástrica, cólicos, chistata, infección renal, dolor menstrual, inflamación, ulcera }\end{array}$ \\
\hline OREGANO & & $\begin{array}{l}\text { C. Amboinicus L graveolens Indigestión, tos Bronquitis, etc. Debido a su contenido de } \\
\text { Alcanfor, en cantidades grandes, puede causar trastornos. }\end{array}$ \\
\hline $\begin{array}{l}\text { PICODE } \\
\text { PÁJARO }\end{array}$ & & $\begin{array}{l}\text { Fiebre, catarro, tos, tosferina, dolor de garganta, dolor estómago, diarrea, colitis } \\
\text { aguda, cólicos, higado, ictericia, dolor menstrual, Occidentalis C. Occidentalis } \\
\text { Conjuntivitis, cólicos. La semilla contiene una substancia que la hace completamente } \\
\text { tóxica para el ganado. Quelite de Fraile J. Gossyplifolia L. Afecciones Renales, cólicos, } \\
\text { etc. Las semillas por su contenido de Curcinaes bien tóxica. Los sintomas de } \\
\text { intoxicación son quemaduras, vasca, diarrea. }\end{array}$ \\
\hline SÁBILA & & $\begin{array}{l}\text { Para la asma, indigestión, gastritis, ulceras digestivas, estreñimiento, higado, mal orin, } \\
\text { hemorroides, inflamación, alergia, erisipela, caida del cabello, ulceras varioosas, } \\
\text { repelente contra insectos, ayuda con las quemaduras leves. }\end{array}$ \\
\hline $\begin{array}{l}\text { UNADE } \\
\text { GATO }\end{array}$ & & $\begin{array}{l}\text { La planta se llama así por los pares de espinas grandes, encorvadas que crecen a lo } \\
\text { largo. Su nombre cientifico es Uncaria tomentosa. Se usan tradicionalmente el ladrido } \\
\text { interno y la raiz para hacer un tė. Trata las enfermedades inflamatorias como la artritis, } \\
\text { para limpiar el tracto digestivo y para tratar el cáncer. También se ha usado para tratar } \\
\text { la disenteria, la recuperación del parto, y los desequilibrios de la hormona de mujeres. } \\
\text { Normaliza algunas funciones del sistema inmune. También parece ayudar a reducir la } \\
\text { coagulación de sangre. }\end{array}$ \\
\hline
\end{tabular}




\section{Cuadro No2. Plantas medicinales no nativas de la comunidad de Chagüitillo}

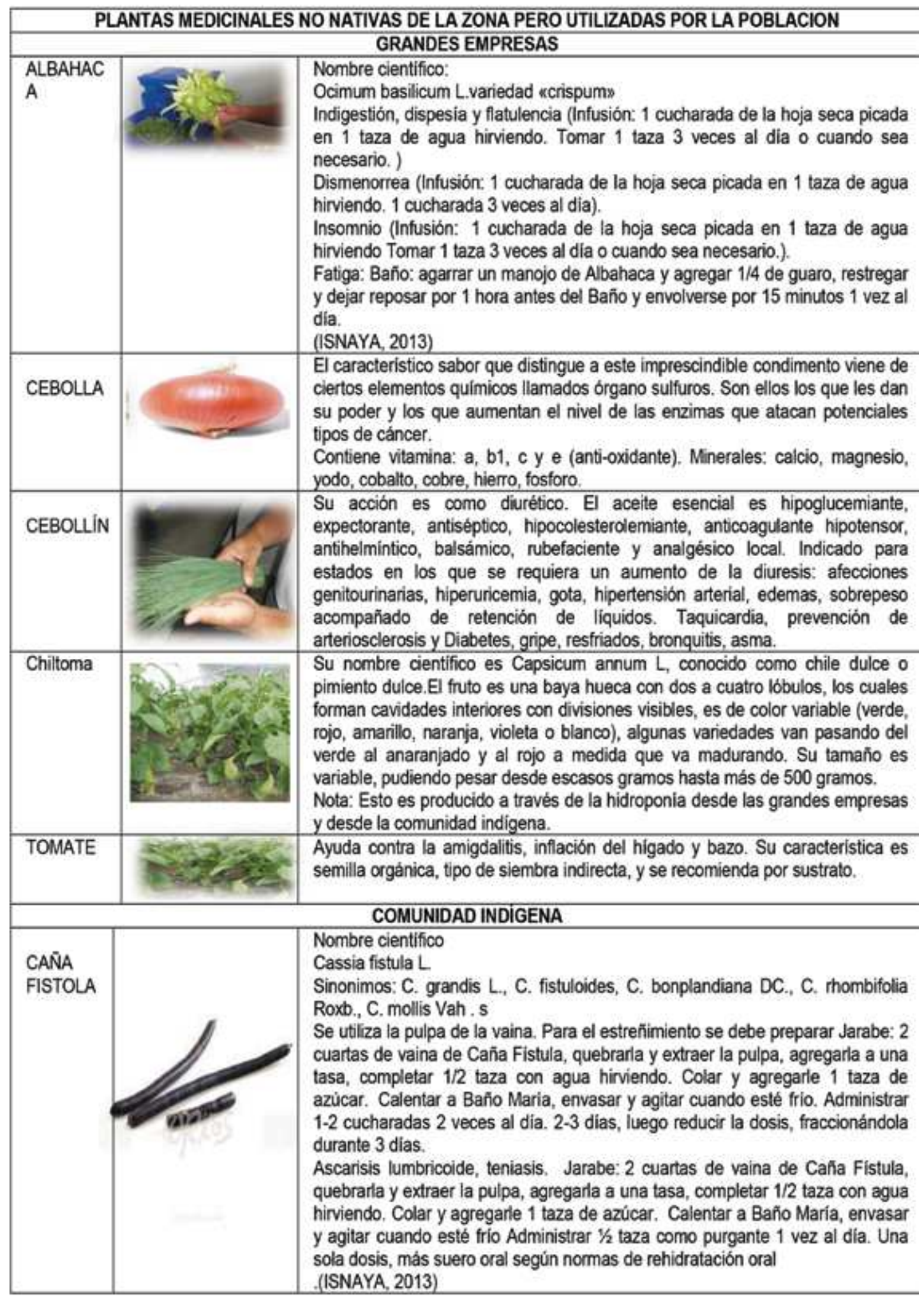




\section{Discusion de los Resultados}

El conocimiento utilitario y conocimiento tradicional que la población posee sobre las plantas medicinales se caracteriza desde la cultura material e inmaterial.

Según Celso Meza Escobar, las plantas medicinales aparte de tener un valor curativo en la medicina tradicional, también tiene un valor cultural y comercial. En el caso medicinal la constitución de Nicaragua ha promovido el aprovechamiento de la medicina tradicional, para asegurar el acceso efectivo a los servicios de salud de las comunidades indígenas.

En la transmisión del conocimiento tradicional sobre las plantas medicinales, se identifica el papel de la etnoecología que pone el acento en la sabiduría de las poblaciones versus los conocimientos científicos. En la población de Chagüitillo se identifica que los saberes locales son sistemas de conocimiento holísticos, acumulativos, dinámicos y abiertos, que se construyen con base en las experiencias locales trans-generacionales $\mathrm{y}$, por lo tanto, en constante adaptación a las dinámicas tecnológicas y socioeconómicas; esa sabiduría se demuestra tanto en la cultura material como inmaterial. La cultura material, porque se utilizan diversas partes de las plantas como madera por ejemplo, y cultura inmaterial, porque se utilizan para remedios, para curar diversos males, apartar espíritus, también se les asigna poder, curación, fe y simbolismo.

Las plantas medicinales de Chagüitillo al ser identificadas y percibidas como recursos materiales, permite identificar en la población un conocimiento utilitario, cosmovisiones de los recursos naturales. Con ello se adapta una visión que restringe la idea de lo utilitario a lo meramente material, es decir la investigación basada en el caso de las plantas, las cuales desde la perspectiva utilitaria pueden aparecer a toda cultura como irrelevantes, significantes, protegidas o cultivadas.

Las hierbas medicinales y las plantas aromáticas como el apio, el perejil, la albahaca, el orégano, el tomillo el cebollín, son especies que se han cultivado muy bien mediante esta técnicas y muy promisorias en los mercados internacionales tanto para consumo fresco como para la obtención de extractos y esencias, por tal razón es que inversionistas extranjeros(Israel) asociados con empresarios Nicaragüenses y debido a las condiciones climáticas de la comunidad indígenas, han abierto "zonas francas", como ellas lo denominan.

La población al producir plantas medicinales lo ha hecho a base del uso de la tierra, pero desde hace siete año se apropiaron de la innovación de la hidroponía lo cual demuestra que dicha forma de producir plantas medicinales se ha construido a través de las memorias colectivas, adaptando dinámicas tecnológicas. La naturaleza, cultura y producción son entonces, aspectos que permiten la construcción de los saberes locales, mismos que se basan en las experiencias individuales y sociales desarrolladas y utilizadas por el productor rural en el acto de realizar la apropiación de la naturaleza clasificando el uso de las mismas desde la cultura no material (ornamental estético, simbólico, religioso, funerario, festivo, protector, histórico, mágico y mítico) y la cultura material.

Las cosmovisiones indígenas que se encuentran en Chagüitillo, sobre los elementos que permiten los procesos de producción de plantas medicinales, permite identificar a través del conocimiento dinámico (fenómenos o 
procesos de la naturaleza). En este ámbito se reconocen los conocimientos que los productores rurales tiene sobre diferentes fenómenos de la naturaleza tales como ciclos lunares, movimientos de materiales sobre la superficie, eventos climáticos, ciclos de vida de las especies y periodos de floración, todos los cuales adquieren una enorme importancia en la ejecución de los procesos productivos. Todos los conocimientos antes descritos, perviven por la sabiduría local, la cual se basa en la experiencia concreta y en las creencias compartidas por los individuos acerca del mundo circundante, mantenida mediante testimonios.

\section{Producción de Plantas Medicinales e Hidroponía}

Desde el análisis cuantitativo, se aborda quienes son los que hacen uso de las plantas medicinales.

El siguiente gráfico $\mathrm{N}^{\circ} 1$ (equivalente al número 2 en el Anexo D), es resultado de la aplicación de (27) veintisiete encuestas realizadas a la población a través de las encuestas con Escalas de Likert.

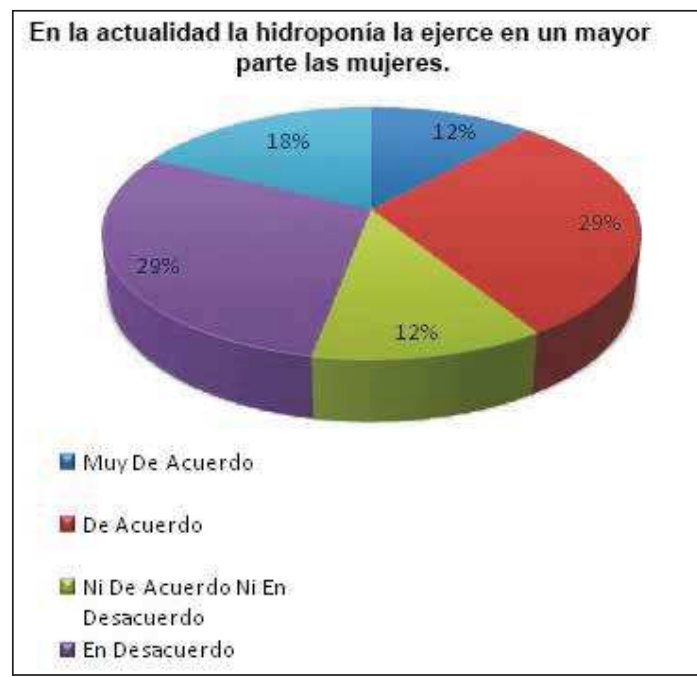

Gráfico $\mathbf{N}^{\mathbf{0}} \mathbf{1}$ Uso de la hidroponía por parte de la población
En el presente gráfico $\mathrm{N}^{\circ} 1$, se afirma según los encuestados, que la mayor parte de la población que utiliza hidroponía a gran escala no son las mujeres, con un total de $47 \%$ en desacuerdo, a diferencia de un $42 \%$ que afirma que las mujeres son las que mayor ejerce la hidroponía.

A través de las encuestas aplicadas, se solicitó a las personas que emitieran su criterio acerca de la importancia de la hidroponía como un método de protección del medio ambiente, ya que no daña el suelo y ahorrar agua en la cosecha, lo cual se refleja en el gráfico $\mathrm{N}^{\circ} 2$ siguiente:

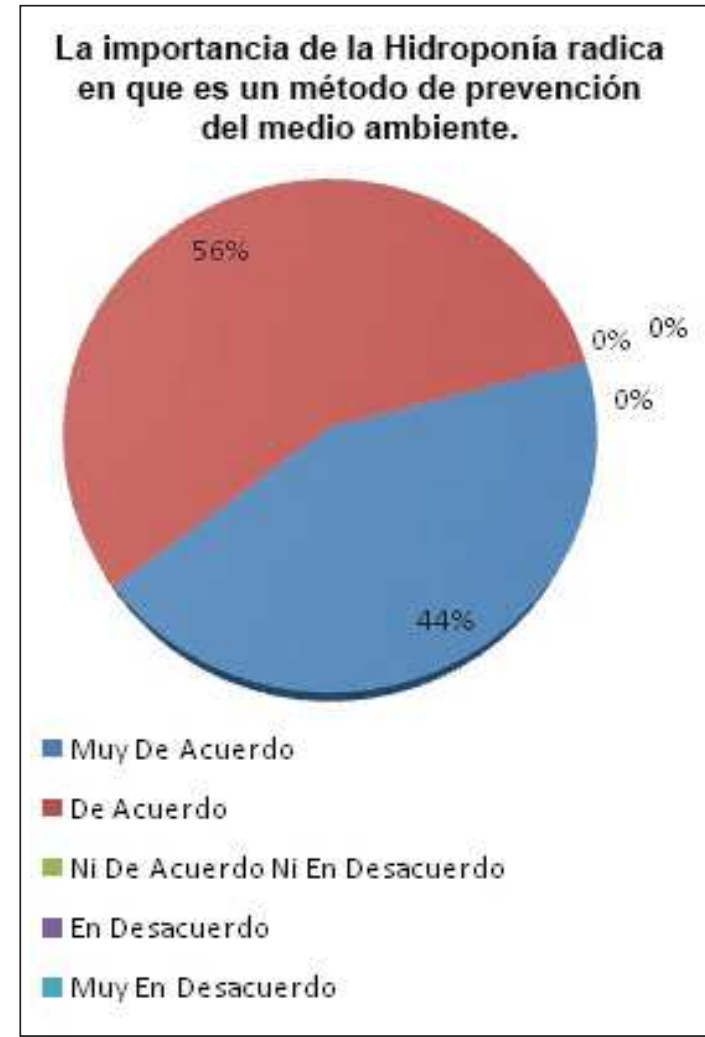

Gráfico $\mathbf{N}^{\circ}$ 2. La importancia de la Hidroponía

El gráfico $\mathrm{N}^{\circ} 2$ (equivalente al $\mathrm{N}^{\circ} 3$ en el Anexo D), muestra que de un total de veintisiete encuestados, están totalmente de acuerdo que la importancia de la 
hidroponía radica en ser un método de protección del medio ambiente.

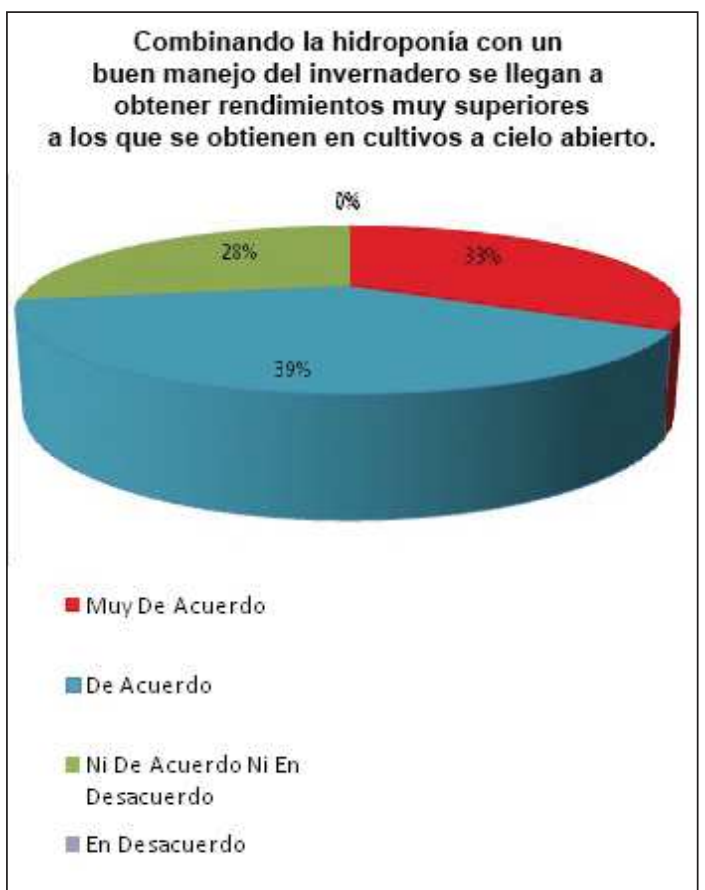

Gráfico $\mathbf{N}^{0} 3$. Ventajas de la Hidroponía

En el gráfico $\mathrm{N}^{\circ} 3$ (equivalente al $\mathrm{N}^{\circ} 6$ en el Anexo D) se muestra que de un total de veintisiete encuestado, el $72 \%$ de la población está de acuerdo que con la hidroponía se puede llegar a obtener rendimientos muy superiores a los que se obtienen en cultivos a cielo abierto, siendo esta una gran ventaja.

La creación de huertos de plantas medicinales hidropónicos en la comunidad de Chagüitillo muestra una sustentabilidad ecológica de largo plazo en lugar de la productividad de corto plazo. Los entrevistados plantean que se debe mejorar el sistema de producción reduciendo el uso de recursos (caso del agua), la pérdida de nutrientes por escorrentía, y mejorar el reciclado de nutrientes, mediante la utilización abonos orgánicos.

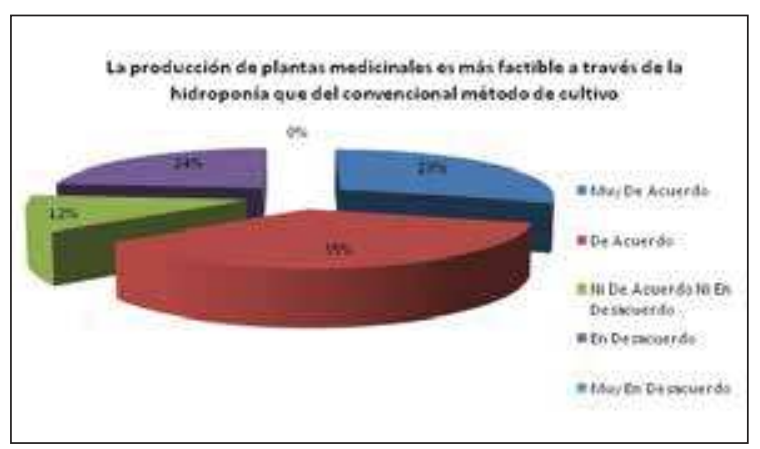

Gráfico $\mathbf{N}^{\circ}$ 4. Cultivo Hidropónico vs. Cultivo tradicional.

El gráfico $\mathrm{N}^{\circ} 4$ (Equivalente al $\mathrm{N}^{\circ} 10$ del Anexo D) muestra que de un total de veintisiete encuentras, el $64 \%$ está de acuerdo que la producción de plantas medicinales es más factible a través del método hidropónico que del método tradicional.

En otro aspecto, existen tres criterios característicos de la sustentabilidad en el cultivo hidropónico de las plantas medicinales de Chagüitillo, como son: uno la conservación de ecosistemas, dos de culturas tradicionales y locales y tres conservación de plantas medicinales.

La población plantea que existen factores potenciales para la utilización de hidroponía a nivel de los huertos familiares a pequeña escala, donde se cultivan rubros que son parte de la dieta familiar..

La experiencia hidropónica de Chagüitillo plantea factores que potencian la producción y otros no favorables para el cultivo de plantas medicinales en huertos. Las opiniones fueron planteadas por la población indígena reunida en el Taller de Validación 2013 (Ver anexo F), realizada en el mes de abril. Los factores no favorables son: el poco acceso al agua, mal uso de nutrientes, desconocimiento de la cantidad 
de sustrato, y la idea que para producir se necesitan grandes conocimientos agronómicos para su manejo. Entre los factores potenciales están: presencia de luz al menos seis horas al día, la temperatura y costo de mantenimiento barbato. (Ver anexo I)

La población, además planteaba que las limitaciones de suministro de agua (2 horas de abastecimiento diariamente) no permiten aumentar el área de huertos familiares hidropónicos, ya que se necesitan grandes cantidades de agua para la producción. Esta dificultad podría afectar las posibilidades de la disponibilidad de alimentos para las familias y por tanto la seguridad alimentaria.

Al conocer esta problemática uno de los propósitos de este trabajo fue poder capacitar a la población sobre la producción de plantas medicinales con hidroponía. Ante el planteamiento de la necesidad de grandes cantidades de agua, se presentó en el taller una propuesta de solución que consiste en la utilización de fibra de coco.

El otro planteamiento consistió en la creencia de que se requiere una alta inversión para implementar la tecnología hidropónica, ya que se desconocía que existen métodos artesanales para la producción de plantas medicinales con hidroponía.

El conocimiento de la técnica hidropónica está relacionado con familias que han tenido vínculos con las empresas productoras hidropónicas, y por esa razón se sostiene que la mayoría de las personas no cultivan por hidroponía sobre todo aquellas que habitan en comunidades rurales. Las familias que sí poseen huertos a base del método hidropónico tienen la característica que al menos uno de sus miembros aprendió el sistema después de establecer una relación laboral con las empresas hidropónicas. Por ejemplo, el esposo de la profesora Martha Ortiz Gallardo, aplicando los conocimientos que adquirió en la empresa, estableció en su patio un huerto familiar en el que se produce plantas medicinales: albahaca y ruda; y además alguna hortaliza como la chiltoma.

Otro caso es el del señor Vicente Palacios, quien trabaja en los invernaderos de la Empresa Hidropónica de Nicaragua, desde hace siete años, estableció su huerto familiar con método hidropónico. Entre los rubros que produce se puede mencionar: albahaca como planta medicinal, pero también cultiva hortaliza y verduras tales como: tomate, ayote y repollo, las cuales poseen beneficios medicinales y nutricionales, además señala que los cultivos producidos a base de hidroponía son sanos y libre de contaminación. Don Vicente utiliza las cosechas de los rubros para autoconsumo.

En relación al mantenimiento de los cultivos participa toda la familia, quienes conocen la técnica y manejo a pequeña escala. La tecnología incluye el uso de raíz en sustrato sólido (arenas, gravilla, escoria de carbón, ladrillo molido, piedra pómez, cascarilla de arroz, aserrín, viruta de madera, arcilla expandida, vermiculita, lana de roca.) En el caso del señor Palacios utiliza la fibra de coco y cascarilla de arroz.

\section{Conclusiones}

Al analizar la producción de plantas medicinales con el uso de cultivos hidropónicos, como alternativa a la conservación de la medicina tradicional desde un enfoque antropológico, se concluye que las personas si consumen plantas medicinales pero que del $100 \%$ de la población el $40 \%$ utiliza el método de 
cultivo hidropónico para la producción de plantas medicinales.

Los mayores usuarios del método hidropónico, para la producción, son las zonas francas "Hidropónica de Nicaragua S.A y Valley Fresh S.A", las cuales exportan grandes cantidades, a los Estados Unidos y Canadá.

Las características sociales, económicas, políticas y culturales de la población de Chagüitillo, Sébaco- Matagalpa, están bajo la cosmovisión indígena, poseen consejo de anciano y casta indígena, los cuales son los que ordenan en la comunidad, dejando en segundo plano a los CPC. Además se encuentra la asociación $\mathrm{ADCH}$, que por medio de su gestión ha llevado proyectos en los que se ha beneficiado la comunidad en distintos ámbito.

En el aspecto cultural, Chagüitillo se caracteriza por su santo patrono San Juan, cuya fiesta es el 24 de junio. Además en la zona existe lo conocido como el santuario de los venados, un lugar donde se encuentran un sinnúmeros de plantas medicinales y petroglifos cuyo mantenimiento está a cargo del Museo Precolombino de Chagüitillo.

El conocimiento tradicional, la experiencia y la sabiduría forman la base de la etnobotánica que incide en la aplicación de los saberes con fines utilitarios, dinámicos y relacionales, especialmente en cuanto a la producción de plantas medicinales con base en el método hidropónico. Esta innovación ha generado un impacto positivo y ecológicamente amigable del entorno ambiental y la economía de las/ los productoras/es.

\section{Referencias Bibliográficas}

Antillon, L. A. (2006). Hidroponia Cultivo sin tierra. Costa Rica: Ed.

Blanco. (2008). Tejo y cultura: de la tradicion etnobotanica a la farmacologia cientifica.

Muños, F. (1996). Plantas medicinales y Aromaticas. Mexico.

TBlanco, E. (2008). Tejo y cultura: de la tradicion etnobotanica a la farmacologia cientifica..

Schultes, r. e. (1990). Etnobotanica. Cordoba.

Toledo, V. M. (1991). El juego de la Supervivencia.Chile: CLADES. 